\title{
Introducing Weather Factor Modelling into Macro Traffic State Prediction
}

\author{
Yuwei Bie, ${ }^{1}$ Tony Z. Qiu, ${ }^{1,2}$ Can Zhang, ${ }^{1}$ and Cunbao Zhang ${ }^{2}$ \\ ${ }^{1}$ Department of Civil and Environmental Engineering, University of Alberta, Edmonton, AB, Canada \\ ${ }^{2}$ Intelligent Transportation Systems Research Center, Wuhan University of Technology, Wuhan, China \\ Correspondence should be addressed to Cunbao Zhang; zhangcunbao@163.com
}

Received 9 September 2016; Revised 22 November 2016; Accepted 19 December 2016; Published 9 February 2017

Academic Editor: Steven I. Chien

Copyright (C) 2017 Yuwei Bie et al. This is an open access article distributed under the Creative Commons Attribution License, which permits unrestricted use, distribution, and reproduction in any medium, provided the original work is properly cited.

\begin{abstract}
Adverse weather has significant impacts on road conditions and traffic dynamics. It is observed that adverse weather as a set of exogenous factors lowers the free flow speed, shifts critical density, decreases flow capacity, and makes the freeway more prone to congestion. This paper proposes a weather factor model to be plugged into a macroscopic traffic prediction model, so that under bad weather traffic variables can be more accurately and reasonably estimated and predicted for traffic control use. To be specific, weather-specific fundamental diagrams are built by introducing weather factors to free flow speed, capacity, and critical density. The weather factors are modelled by selected weather measurements. Weather factor parameters are trained by recent historical weather and traffic data and then can be put into real-time macro traffic prediction and control. The traffic prediction model in the case study is METANET model, in which fundamental diagram parameters are one source of input. The weather-specific prediction error and conventional prediction error are compared. Real data collected by loop detectors on Whitemud Drive, Edmonton, Canada, is used for parameter calibration and prediction error evaluation. The results show that the proposed weather models reasonably improved the accuracy of macro traffic state prediction model compared to conventional model.
\end{abstract}

\section{Introduction}

Over the past two decades, the focus of efforts in modelling and forecasting macroscopic traffic states has transitioned from univariate temporal correlation to multivariate temporal-spatial correlation and from linear to nonlinear forms. Those models may be loosely classified as statistical and nonstatistical methods. Some examples are included in the class of time series models, like the seasonal autoregressive integrated moving average model [1], Kalman filter state-space model [2], neural network [3], nonparametric regression [4], stochastic Newell's three-detector method [5], and other empirical models.

Another class of works is based on the use of macroscopic traffic flow theory to estimate the internal traffic state for any intermediate point on a freeway or arterial segment from the boundary conditions. Macroscopic models consider traffic flow as fluid instead of individual vehicles. Three variables are capable of describing traffic stream characteristics: flow, density, and mean speed. Macroscopic traffic flow models are classified as first-order, second-order, or higher-order models, depending on the number of differential equations included [6]. Of all first-order models, the most used one is the Lighthill-Whitham-Richards model $[7,8]$, which uses one partial differential equation to describe the vehicle flow conservation law. This model was also the first combination of a traffic flow model with a static fundamental diagram. Another representative first-order model is the Cell Transmission Model [9-11], which is a discretized and simplified version of the Lighthill-Whitham-Richards model. The Payne model [12] is the oldest second-order traffic flow model. Besides the flow conservation law equation, the Payne model also includes one partial differential equation that describes mean speed dynamics. This model can replicate traffic phenomena with higher accuracy. Other types of second-order traffic flow models include variation kinematic waves [13], second-order traffic flow model with Kalman filter [14], CTM-based second-order traffic flow model with particle filtering [15], the Lighthill-Whitham-Richards partial differential equation with the Lagrangian measurements 
[16], and Newell's simplified kinematic wave model [17, 18]. The second-order traffic flow model selected in this paper is METANET $[19,20]$, which is a discretized and improved version of the Lighthill-Whitham-Richards model combined with the Payne model. The reason to choose METANET model is that it has three separate dynamic functions to predict traffic flow and average speed and density [21]. It has a discrete space-time form and allows for convenient discretization intervals [6], so that field collected data can be easily implemented. The METANET model has nice and simple analytical properties: it has an explicit state-space form and contains continuous and differentiable functions [6]. The clear mathematical form of METANET model allows the introduction of other methods such as Kalman filtering, optimization, and weather models. This model can also be used for optimal real-time control of freeway traffic such as variable speed limit and ramp metering [22]. In this paper, the macroscopic traffic state prediction will be the METANET model.

Weather affects many aspects of road conditions. Maze et al. provided evidence that traffic demand, safety, operations, and flow can be reduced by rain, snow, fog, cold, and wind [23]. Methods based on aggregated flow and speed measurements from local sensors have been used to estimate capacity and free flow speed during adverse weather [24]. HCM (2010) volume 1 chapter 5 provides fixed capacity reduction percentage to estimate the impacts of bad weather on the capacity of urban roads [25]. What HCM (2010) provided does not consider the weather features in different countries such as Canada or in real time. And those reduction percentages cannot be applied to traffic features other than capacity, such as free flow speed or critical speed. Kwon et al. showed that in many cases the HCM underestimate or overestimate the real effects [26]. Similar to HCM, FHWA (2004) provided a report giving fixed weather impact factors by simulating different weather conditions [27]. FHWA (2006) showed empirical studies and statistics about the impact of inclement weather on roadways [28]. Alhassan and Ben-Edigbe conducted an empirical analysis of highway capacity loss due to rainfall [29]. Hou et al. proposed that in mesoscopic network simulation weather factors can be introduced in the calibration of the traffic flow model for adverse weather [30]. Shahdah and Fu simulated the impact of winter weather on road mobility on a Canadian freeway [31]. In 2013, Lam et al. modelled the effects of rainfall intensity on traffic speed, flow, and density relationships and calibrated model using hourly rainfall data from Hong Kong [32]. In this paper, a weather factor modelling method for free flow speed, critical density, and capacity is suggested and calibrated by field data. The proposed model is able to estimate the impact of snowy Canadian weather in real time and can be potentially implemented in traffic prediction and control.

The weather factor model is developed and then can be inserted into the traffic state prediction model using field data. Building on previous work, our study has three contributions. Firstly, more than one weather factor are considered and filtered under weather conditions varying from the worst to the best weather and then applied to different macroscopic traffic variables. In contrast, previous works consider only one category of extreme weather and one traffic variable. Secondly, weather factors are successfully introduced into a fundamental diagram as well as a traffic prediction model. Thirdly, this paper uses high-resolution field data instead of simulation data to obtain clear quantitative results that show weather significantly impacts the traffic dynamics on freeways.

The rest of this paper is organized as follows. The macro traffic state prediction model is described in Section 2, highlighting the modification for weather conditions. The definition and calibrating skills for fundamental diagram are also described in Section 2. The modelling of weather factors to be applied to fundamental diagrams is studied in Section 3. In Section 4, in case study the proposed weather factor model is calibrated and applied to traffic prediction model using field collected data, and the effect on improving prediction accuracy is shown. Section 5 concludes the paper.

\section{A Macro Traffic State Prediction Model Factoring in Road Conditions}

The model description is separated into four parts. Section 2.1 will provide notations and assumptions. Sections 2.2 and 2.3 will present the description of the traffic state prediction model and the description of the fundamental diagram (FD) used in this study. Section 2.4 will describe the model calibration methods.

2.1. Notations and Assumptions. For consistency, the freeway is divided into $N$ sections with lengths $\Delta_{i}, i=1, \ldots, N$, each having at most one on-ramp and off-ramp. All the variables used throughout this paper are defined as in the Notations.

The assumptions of this paper are listed as follows. (1) In the case study part, field data collected by loop detectors will be used. This paper assumes that loop detector data can be regarded as ground-truth. (2) The weather data used in case study is collected by one weather detection station in Edmonton city. Since the case study freeway segment is within $1 \mathrm{~km}$ radius from the weather station and the snow on freeway is not removed promptly, the authors of this paper assumed that weather station detected snow depth is proportional to the snow depth on freeway road surface, so that it is reasonable to use weather station detected data in this study.

2.2. METANET Prediction Model Framework. The development of a mathematical model that describes the dynamic evolution of three traffic variables enables the short time prediction of macroscopic traffic states. The METANET model has three dynamic functions that represent flow, density, and speed. Among the three dynamics, the flow dynamics and density dynamics are exact analytical models without parameter calibration, and are derived from the flow conservation law $[33,34]$ written as follows:

$$
\frac{\partial \rho(t, x)}{\partial t}+\frac{\partial q(t, x)}{\partial x}=r(t, x)-s(t, x) .
$$


This conservation law equation indicates that the vehicle entering one section will eventually exit, moving onto either the next section of main road or the off-ramp. The flow and density dynamics together are the first-order part of the METANET model. However, with solely first-order dynamics the dynamics of speed change cannot be described. Although there is a certain relationship between speed and density, speed does not change instantaneously as density changes in the real world. Payne proposed that a small time delay should be applied to the speed-density relationship in the FD as follows:

$$
v(x, t+\tau)=V[\rho(x+\Delta x, t)] .
$$

Papageorgiou et al. [19] expanded the left-hand side of the above equation in a Taylor series with respect to $\tau$ and the right-hand side with respect to $\Delta x$; after rearranging the terms, the following equation is obtained:

$$
\tau \cdot \frac{\partial v}{\partial t}=V(\rho)-v-\frac{v}{\rho} \cdot \frac{\partial \rho}{\partial x},
$$

where $\mathrm{d} v / \mathrm{d} t$ is the acceleration rate of an observer moving with the traffic stream [19]. $\mathrm{d} v / \mathrm{d} t$ is written as follows:

$$
\frac{\mathrm{d} v}{\mathrm{~d} t}=\frac{\partial v}{\partial t}+v \cdot \frac{\partial v}{\partial x}
$$

Substituting (3) into (4) results in the continuous form of speed dynamics model

$$
\frac{\mathrm{d} v}{\mathrm{~d} t}=v \cdot \frac{\partial v}{\partial x}+\frac{[V(\rho)-v-(v / \rho) \cdot(\partial \rho / \partial x)]}{\tau} .
$$

Combining (1), (5), and the identical equation $q=\rho$. $v$ together forms the three dynamics of the METANET model. With the introduction of the speed dynamics model, METANET is able to predict macroscopic traffic states accurately. The discretized model formulation is written as follows.

Density dynamics are

$$
\begin{aligned}
\rho_{i}(k+1)= & \rho_{i}(k) \\
& +\frac{T}{\Delta_{i}}\left[q_{i-1}(k)-q_{i}(k)+r_{i}(k)-s_{i}(k)\right] .
\end{aligned}
$$

Speed dynamics are

$$
\begin{aligned}
v_{i}(k+1)= & v_{i}(k)+\frac{T}{\tau}\left[V\left(\rho_{i}(k)\right)-v_{i}(k)\right] \\
& +\frac{T}{\Delta_{i}} v_{i}(k)\left[v_{i-1}(k)-v_{i}(k)\right] \\
& -\frac{\eta \cdot T\left[\rho_{i-1}(k)-\rho_{i}(k)\right]}{\tau \cdot \Delta_{i} \cdot\left[\rho_{i}(k)+\kappa\right]} .
\end{aligned}
$$

Flow dynamics are

$$
q_{i}(k+1)=\rho_{i}(k+1) \cdot v_{i}(k+1) .
$$

In the model $\tau$ (seconds) and $\kappa(v e h / \mathrm{km})$ and $\alpha$ and $\eta$ $\left(\mathrm{km}^{2} / \mathrm{h}\right)$ are global model parameters to be calibrated using the historical data. The parameter $\tau$ (seconds) describes the gradient of current speed approaching desired speed. The larger $\tau$ (seconds) is, the larger the difference between current speed and desired speed is. In practice, a large $\tau$ value can improve the prediction accuracy through tuning effects, however, since $\tau$ has physical meaning as a lag time item, the biggest acceptable value of $\tau$ is set as $120 \mathrm{~s}$ empirically, meaning that a tolerance time of $120 \mathrm{~s}$ is allowed for vehicles to adjust their speed to desired speed in general road conditions. The constant $\kappa>0$ is added to keep the fourth term of (7) limited when density is low. Again $\kappa$ is empirically fixed as $10(\mathrm{veh} / \mathrm{km})$, since $\kappa$ is put together with $\rho_{i}(k)$ which typically fall into a range of $0 \sim 50(\mathrm{veh} / \mathrm{km})$. The fixing of $\kappa$ does not impact the prediction accuracy, because $\eta$ and $\kappa$ together make up the parameters of the fourth term of (7). $\eta$ is calibrated using training dataset and usually falls within $0 \sim 100\left(\mathrm{~km}^{2} / \mathrm{h}\right) . \alpha$ usually falls within $2 \sim 4$. The desired speed $V\left(\rho_{i}(k)\right)(\mathrm{km} / \mathrm{h})$ in the speed dynamics model is represented by

$$
V\left(\rho_{i}(k)\right)=v_{f, i} \exp \left[-\frac{1}{\alpha}\left(\frac{\rho_{i}(k)}{\rho_{\mathrm{cr}, i}}\right)^{\alpha}\right] .
$$

The desired speed variable leaves room for the introduction of weather factors because it involves two important parameters calibrated from the FD: free flow speed and critical density, and the FD is impacted by weather. Density dynamics do not involve parameters and other parameters in speed dynamics are calibrated globally for goodness of fit. Note that, of the four terms making up the speed dynamics, each term has a physical meaning. In (7), the second term is referred to as the relaxation term, describing that, with a lag time item $\tau$, the mean speed $v$ of the link gets relaxed to the desired speed, which largely depends on the parameters of the FD. In this study, we chose the format of desire speed as (9). The third is the convection term, indicating that vehicles travelling from upstream link $i-1$ to current link $i$ adapt their speed gradually rather than instantaneously. The fourth is the anticipation term, which is to indicate that drivers are always watching the traffic condition on the road ahead. For example, if a driver observes high traffic density in the downstream link $i+1$, the driver then reacts by slowing down. Taking into account weather-specific FDs, it can be concluded that the desired speed variable is the right place to insert the weather factor.

The Courant-Friedrichs-Lewy (CFL) condition should be followed, which means to ensure that vehicles cannot travel beyond one section within computing time interval $T$, so that $T$ satisfies the following condition:

$$
T \leq \frac{\Delta_{i}}{\max \left(v_{f, i}\right)}, \quad i=1,2, \ldots, N .
$$

2.3. The Weather-Specific Fundamental Diagrams. It is assumed that the FD changes according to different weather conditions, since weather significantly impacts driver behavior and the driving environment. The weather-specific 


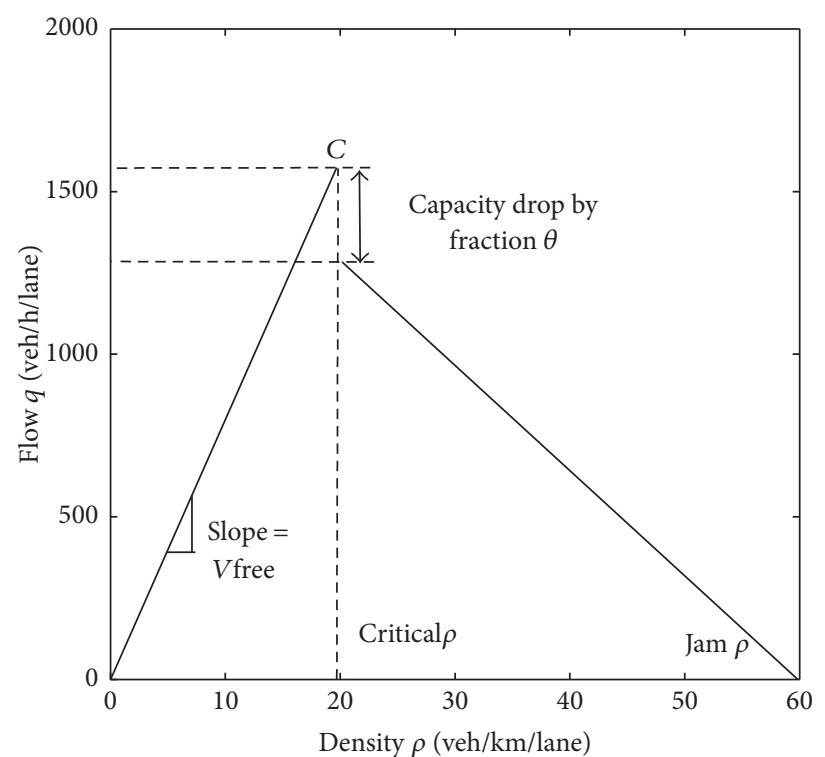

(a)

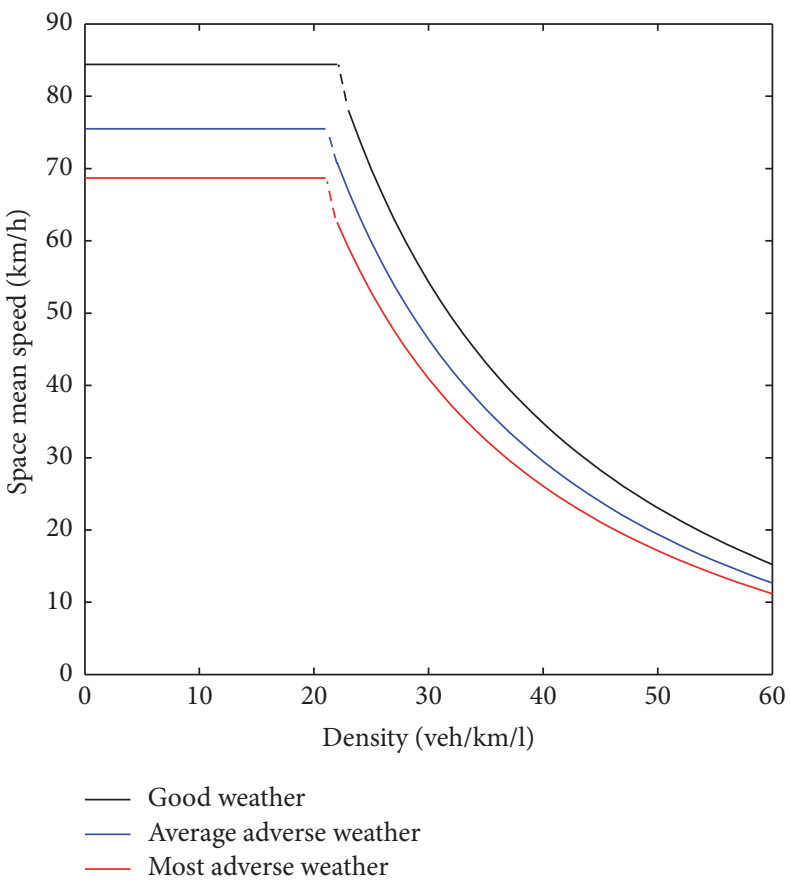

(b)

FIgURE 1: Illustration of (a) triangular FD and (b) its variation with speed as the vertical coordinate.

FD defines different $V\left(\rho_{i}(k)\right)$ for different weather conditions in the METANET model. To be specific, the parameters of FD such as free flow speed, critical density, jam density, and capacity drop vary with weather conditions instead of being constants as regarded before. And those key parameters in the FD are essential for the accuracy of the traffic prediction model. The triangular FD is still used here shown as Figure 1(a). Note that, to better exhibit the different free flow speeds under different weather conditions, we also demonstrate the variation form of the triangular FD, for which the vertical coordinate is space mean speed as Figure 1(b) shows. The three weather conditions are defined as "good weather" which means no snow; "average adverse weather" which means $1-15 \mathrm{~cm}$ of snow; and "most adverse weather" which means more than $15 \mathrm{~cm}$ of snow. The definition is based on Edmonton, Canada, and will be used in case study.
From the point of the space mean speed, the triangular FD describes that, before density reaching critical density, space mean speed keeps in free flow speed. After reaching critical density, traffic becomes congested and the road segment capacity drops by fraction $\theta$ due to the unsatisfying driving environment. After reaching the congestion point, traffic flow decreases linearly to zero, which is when density also reaches maximum, and that density is referred to as jam density. In the variation form of the triangular FD, space mean speed behaves similarly, remaining constant up until the congestion point, and, after that, speed will drop together with capacity. During congestion, the space mean speed will drop as inverse proportional function of density. Figure 1(b) shows that we anticipate that under different weather conditions FD will shift. The expression of the variation form of the FD is as follows:

$$
v_{i}(k+1)= \begin{cases}v_{f, i}, & \text { if } \rho_{i}(k+1) \in\left(0, \rho_{\mathrm{cr}, i}\right) \\ \frac{v_{f, i} \cdot \rho_{\mathrm{cr}, i}}{\rho_{i}(k+1)}\left(1-\theta-\frac{\rho_{i}(k+1)-\rho_{\mathrm{cr}, i}}{\rho_{\mathrm{jam}, i}-\rho_{\mathrm{cr}, i}}\right), & \text { otherwise. }\end{cases}
$$

In (11), weather factors are introduced to adjust the free flow speed, critical density, and jam density of the FD under varying weather conditions. In part 3 of this paper, the modelling of three weather factors will be discussed.
Equation (12) describes the new form of flow dynamics considering capacity drop and weather factors, and this equation will replace the original flow dynamics in the METANET model. 


$$
q_{i}(k+1)= \begin{cases}\rho_{i}(k+1) \cdot v_{i}(k+1), & \text { if } \rho_{i}(k+1) \in\left(0, \rho_{\mathrm{cr}, i}\right) \\ \min \left(\rho_{i}(k+1) \cdot v_{i}(k+1),(1-\theta) \cdot v_{f, i} \cdot \rho_{\mathrm{cr}, i}\right), & \text { otherwise. }\end{cases}
$$

\subsection{Fundamental Diagram and METANET Calibration Meth-} ods. The parameter estimation of the FD is based on data collected by conventional loop detectors. For the parameter calibration of FDs, free flow speed $v_{f, i}$, critical density $\rho_{\text {cr }, i}$, capacity $C_{i}$, jam density $\rho_{\mathrm{jam}, i}$, and capacity drop fraction $\theta$ must be estimated. For calibration, the data format we use is $\left(\rho_{i}, q_{i}\right)$ data points, where the horizontal coordinate is density and the vertical coordinate is flow. The procedure of calibrating a triangular FD is as follows.

Step 1 (The identification of $\rho_{\mathrm{cr}, i}$ and capacity $C_{i}$ ). In defining the triangular $\mathrm{FD}$, the summit point of the triangle roughly indicates critical density and capacity. We plot all $\left(\rho_{i}, q_{i}\right)$ points and find the third largest $q_{i}$ and take it as capacity $C_{i}$ and the corresponding $\rho_{i}$ as $\rho_{\text {cr }, i}$. The reason for not choosing the largest flow is that normally the largest flow is extremely high as an outlier; this might be due to detection error.

Step 2 (The identification of $v_{f, i}$ ). After defining $\rho_{\mathrm{cr}, i}$, the whole dataset can be divided into two parts: the left-side triangle represents uncongested traffic conditions and the right-side triangle represents congested traffic conditions. Then we calculate the slope of each data point distributed in the left side and take an average as $v_{f, i}$.

Step 3 (The identification of $\rho_{\text {jam }, i}$ and capacity drop fraction $\theta$ ). The jam density $\rho_{\text {jam, } i}$ represents a theoretical value of when the road section is totally congested and all vehicles have stopped moving. When determining the rightside triangle, we usually fix $\rho_{\mathrm{jam}, i}$ with an empirical value $100 \mathrm{veh} / \mathrm{km} / \mathrm{l}$. In this study, the empirical value is set according to historical observation of congested situations during winter on the testbed. The slope of the right side is determined by least square fit. After determining the foot and slope of right side, the intersecting point of the right side and the vertical auxiliary line passing through previous capacity $C_{i}$ is the new capacity after dropping.

For the parameter estimation of the METANET model, $\tau, \kappa, \alpha$, and $\eta\left(\mathrm{km}^{2} / \mathrm{h}\right)$ are global model parameters to be calibrated using recent historical data. In this study, the global parameters of the METANET model are fixed, which are calibrated before this study, using loop detector data of the same road segment via the following expression. All variables with the subscript of obs represent field observed data:

$$
\begin{aligned}
\Theta^{*} & =\arg \min _{\Theta}\left[\sum_{i i}^{N}\left\{v_{\text {obs }}^{i i}-f\left(v^{i i} \text { obs } \mid \Theta\right)\right\}^{2}\right. \\
& \left.+\sum_{i i}^{N}\left\{\rho_{\text {obs }}^{i i}-f\left(\rho_{\text {obs }}^{i i} \mid \Theta\right)\right\}^{2}\right]
\end{aligned}
$$

where $\Theta$ is the set of global parameters $\tau, \kappa, \alpha$, and $\eta$. $i i$ is the index of training data points. The set $\Theta^{*}$ represents the specific parameter set that minimize the summation of square of residuals of both speed prediction and density prediction. The parameter calibration is performed by MATLAB in a nested loop fashion, with a gradient of 0.01 . Since $\kappa$ is fixed and empirically $\tau$ obtains the boundary value and $\eta$ and $\alpha$ both have narrow ranges, the computing time is acceptable.

\section{Modelling Weather Impacts on the Fundamental Diagram}

The historical weather data provided by Canadian government website, historical climate data webpage, Edmonton area includes three critical categories of weather data: temperature, amount of snow on the ground, and maximum wind speed. We assume that free flow speed (FFS), capacity, and critical density of the FD of each day are impacted by weather. The three categories of the weather index are capable of expressing most kinds of weather in Edmonton, Canada. People in Edmonton seldom experience rainfall that is heavy enough to influence visibility, and fog is also rare to see; the most common adverse weather is snowy weather. HCM has pointed out that light snow reduces capacity by $5-10 \%$ and heavy snow reduces capacity by $25-30 \%$. From observations and previous data, we assume that ongoing snow may significantly impact FFS. Because the variable "snow on the ground" reflects the mean value at a time point, the variable "derivative of snow on the ground" is added, formulated as below.

$$
\frac{\mathrm{dSG}_{j}}{\mathrm{~d} t_{j}}=\frac{\mathrm{SG}_{j}-\mathrm{SG}_{j-1}}{t_{j}-t_{j-1}}+\varepsilon, \quad \varepsilon \sim \Delta\left(t^{2}\right) .
$$

$\mathrm{SG}_{j}$ is the depth of the snow on the ground for day $j . t_{j}$ is the day and $\varepsilon$ is the error term. In the dataset, $\mathrm{SG}_{j}$ is the depth of the snow, measured in centimeters, corresponding to the area of the city where the target VDS is located.

$$
\mathrm{DSG}_{j}=\frac{\mathrm{SG}_{j}-\mathrm{SG}_{j-1}}{t_{j}-t_{j-1}} .
$$

$\mathrm{DSG}_{j}$ is a derivative of snow on the ground, which is used in our model. We assume that a change of $S_{G_{j}}$ indicates that a weather event is occurring. A positive DSG value means that snow is falling, and a negative DSG value means that the weather is improving and snow is melting. Temp represents temperature and MWS represents maximum wind speed. Weather factors $w^{v_{f}}, w^{\rho_{\text {cr }}}$ and $w^{C}$ are to be introduced to adjust free flow speed, critical density, and capacity of the FD. All three weather factors are ratio of free flow speed, critical density, and capacity in November snowy days to those in 
TABLE 1: Pearson correlation coefficient between multiple factors.

\begin{tabular}{|c|c|c|c|c|c|c|c|}
\hline PCC & SG & DSG & MWS & Temp & $w^{C}$ & $w^{v_{f}}$ & $w^{\rho_{\mathrm{cr}}}$ \\
\hline SG & - & 0.33 & -0.69 & -0.75 & -0.39 & -0.59 & 0.35 \\
\hline DSG & 0.33 & - & -0.30 & -0.50 & -0.75 & -0.74 & -0.12 \\
\hline MWS & -0.69 & -0.30 & - & $\underline{0.54}$ & 0.29 & 0.35 & -0.23 \\
\hline Temp & -0.75 & -0.49 & 0.54 & - & $\underline{0.64}$ & $\underline{0.88}$ & -0.34 \\
\hline$w^{C}$ & 0.39 & -0.75 & 0.29 & 0.64 & - & $\underline{0.73}$ & 0.40 \\
\hline$w^{v_{f}}$ & -0.59 & -0.74 & 0.35 & 0.88 & 0.73 & - & -0.25 \\
\hline$w^{\rho_{\mathrm{cr}}}$ & 0.35 & -0.12 & -0.23 & -0.34 & 0.40 & -0.25 & - \\
\hline
\end{tabular}

TABLE 2: Coefficient $t$-test of weather factors models.

\begin{tabular}{lcccc}
\hline Significance $^{*}$ & SG & DSG & MWS & Temp \\
\hline$w^{v_{f}}$ & YES & YES & NO & NO \\
$w^{C}$ & NO & YES & NO & NO \\
$w^{\rho_{\text {cr }}}$ & NO & NO & NO & NO \\
\hline
\end{tabular}

${ }^{*}$ Significance at $95 \%$ confidence level.

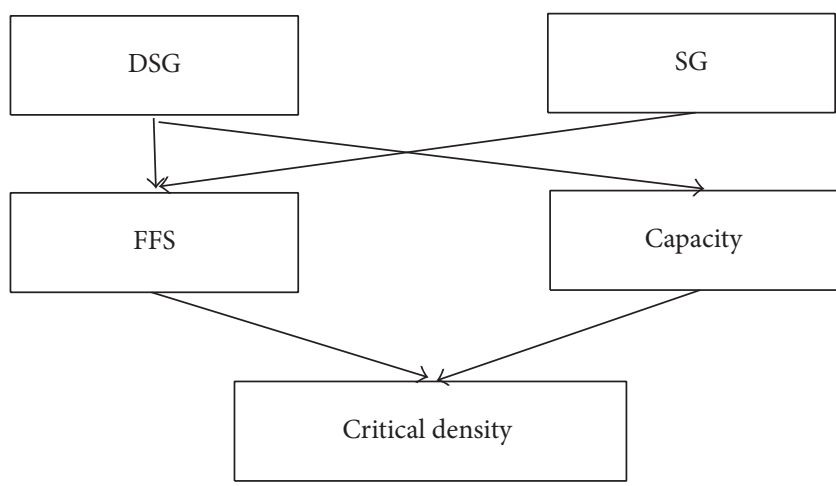

FIGURE 2: Significant weather factors impacting the FD.

May good weather days' average. The weather factors will be modeled by selected combination of independent variables SG, DSG, WMS, and Temp. Field data from one loop detector station in May and November 2013, on Whitemud Drive Edmonton, Canada, is used, and this dataset is the same as that will be used in the following case study part.
After defining all independent and dependent variables, the next step is to filter the key factors impacting FD features. Pearson correlation coefficient (PCC) check (Table 1) is conducted and a $t$-test prior to our case study (Table 2). PCC ranges from -1 to 1 , where 1 is total positive linear correlation, 0 is no linear correlation, and -1 is total negative linear correlation $[35,36]$.

When absolute value of PCC between two factors is over 0.5 which is a normally used threshold, it is then defined that the two factors are highly correlated. It is displayed as underlined value in the table that $w^{C}$ is highly linear related to DSG and Temp and $w^{v_{f}}$ is highly linear related to SG, DSG, and Temp. However, Temp is highly related to both SG and DSG. Hence, DSG is chosen for linear regression of $w^{C}$. And SG and DSG are used for linear regression of $w^{v_{f}} \cdot w^{\rho_{\mathrm{cr}}}$ is not directly correlated to weather variables but can be computed by $w^{C} / w^{v_{f}}$ according to the definition of FD. In Table 2 , we tried the above linear models of $w^{v_{f}}, w^{\rho_{\text {cr }}}$, and $w^{C}$, and the $t$-test of coefficients at $95 \%$ confidence level is shown. This result is identical with that of PCC. Since MWS and Temp seriously confound with SG and DSG, they are not significant as coefficients.

The flow chart in Figure 2 shows how DSG and SG affect FFS, capacity, and critical density in the model of this paper.

The FD under varying weather is formulated as below. Three weather factors, $w_{j}^{v_{f, i}}, w_{j}^{\rho_{\mathrm{cr}, i}}$, and $w_{j}^{C_{i}}$, are introduced to adjust the free flow speed, critical density, and capacity of the FD under varying weather conditions. The capacity adjustment factor $w_{j}^{C_{i}}$ will not appear in the following FD expression, but the critical density adjustment factor $w_{j}^{\rho_{\text {cr,i }}}$ is computed from $w_{j}^{C_{i}}$ as follows:

$$
v_{i}(k+1)= \begin{cases}w_{j}^{v_{f, i}} \cdot v_{f, i}, & \text { if } \rho_{i}(k+1) \in\left(0, \rho_{\mathrm{cr}, i}\right) \\ \frac{w_{j}^{v_{f, i}} \cdot v_{f, i} \cdot w_{j}^{\rho_{\mathrm{cr}, i}} \cdot \rho_{\mathrm{cr}, i}}{\rho_{i}(k+1)}\left(1-\theta-\frac{\rho_{i}(k+1)-w_{j}^{\rho_{\mathrm{cr}, i}} \cdot \rho_{\mathrm{cr}, i}}{\rho_{\mathrm{jam}, i}-w_{j}^{\rho_{\mathrm{cr}, i}} \cdot \rho_{\mathrm{cr}, i}}\right), & \text { otherwise }\end{cases}
$$

so that the flow dynamics equation (12) will also be updated, accordingly, in which

$$
w_{j}^{v_{f, i}}=\beta_{0}+\beta_{1} \mathrm{DSG}+\beta_{2} \mathrm{SG}
$$

$$
\begin{aligned}
w_{j}^{C_{i}} & =\alpha_{0}+\alpha_{1} \text { DSG } \\
w_{j}^{\rho_{\mathrm{cr}, i}} & =\frac{w_{j}^{C_{i}}}{w_{j}^{v_{f, i}}}+\delta=\frac{\alpha_{0}+\alpha_{1} \mathrm{DSG}}{\beta_{0}+\beta_{1} \mathrm{DSG}+\beta_{2} \mathrm{SG}}+\delta,
\end{aligned}
$$


TABLE 3: Fundamental diagram features of target location under the three weather conditions.

\begin{tabular}{lccc}
\hline Features & Good weather condition & Light snow condition & Heavy snow condition \\
\hline$v_{f}(\mathrm{~km} / \mathrm{h})$ & 84.38 & 81.52 & 66.66 \\
$C(\mathrm{veh} / \mathrm{h} / \mathrm{l})$ & 1647 & 1572 & 1323 \\
$\rho_{\mathrm{cr}}(\mathrm{veh} / \mathrm{km} / \mathrm{l})$ & 24.84 & 22.30 & 21.46 \\
$\rho_{\text {jam }}(\mathrm{veh} / \mathrm{km} / \mathrm{l})$ & 100 & 100 & 100 \\
$\theta$ (capacity drop) & 0.05 & 0.09 & 0.17 \\
Sample size & 1440 & 1440 & 1440 \\
\hline
\end{tabular}

TABLE 4: Estimated weather factor parameters and statistics.

\begin{tabular}{lcccc}
\hline & $\alpha_{0}$ & $\alpha_{1}$ & $\beta_{0}$ & $\beta_{1}$ \\
& 0.873 & -0.01796 & 0.9648 & -0.01737 \\
Coefficients with 95\% & $(0.8391,0.9068)$ & $(-0.02908,-0.006853)$ & $(0.9251,1.004)$ & $(-0.02363,-0.0111)$ \\
confidence bounds & $\beta_{2}$ & $\delta$ & & \\
& -0.00105 & 0.1344 & & \\
\hline Adjusted $R$-square & $(-0.00346,0.00136)$ & $w_{j}^{C_{\text {cr, }, i}}$ model: 0.7924 & $w_{j}^{v_{f, i}}$ model: 0.833 & Sample size: 29 \\
\hline
\end{tabular}

where $\beta_{0}, \beta_{1}$, and $\beta_{2}$ are parameters of $w_{j}^{v_{f, i}}$. And $\alpha_{0}$ and $\alpha_{1}$ are parameters of $w_{j}^{C_{i}}$. A constant adjustment factor $\delta$ is added. $i$ is the index of road section, and $j$ is the index of data collection day.

\section{Case Study}

The Vehicle Detection Stations (VDSs) on a 10-kilometre corridor of Whitemud Drive in Edmonton, Canada, collect and store traffic data from dual loop detectors. This section of road plays an important role in people and freight transportation in the city. The road section has two curves and several bottleneck locations where the number of lanes drops. The VDS system currently has 28 VDSs in total, and each station has three or four dual loops. The data recording frequency is 20 seconds. Each dual loop reports the volume $q$ - the number of vehicles crossing the loop detector during a 20-second time interval-and mean speed measurement $v$, as well as the occupancy measurement, which cannot be used directly in traffic control and cannot be transformed accurately into density. The accurate density measurement $\rho$ is calculated by $\rho=q / v$. The target loop detector station is located at a major curve of westbound Whitemud Drive. This target VDS has four dual loops on four lanes, respectively, and is 500 meters from its upstream and downstream VDS.

The calibration and validation of the models are based on the data of target VDSs from May and November of 2013. In this study, three weather conditions are involved in testing the stability of the models. Accordingly, three different FDs and weather-related parameters are generated. In Edmonton, Canada, the main type of adverse weather is snowy weather, so the three weather conditions are categorized as "good weather conditions," "light snow conditions," and "heavy snow conditions." During the days of May 1 to May 5, the snow melt and temperature indicated "good weather conditions." From November 11 to 15, "light snow conditions" were observed, with the snow on the ground measuring around 6-9 $\mathrm{cm}$. During the days of November 18 to 22, "heavy snow conditions" were present, as the amount of snow on the ground ranged $16-26 \mathrm{~cm}$. Figure 3 shows the indexes of the three weather conditions, and Figure 4 shows VDS locations on Whitemud Drive and the location of closest weather station.

Table 3 shows the calibrated FD features and weather factors, while Figure 5 combines data points and the calibrated FD together to help visualize the difference in macroscopic traffic conditions under different types of weather. Under each weather condition, the sample size is 5 days of traffic data aggregated at $5 \mathrm{~min}$ frequency, which means 1440 data points. From the field data we observed that the triangular FD holds. Note that, in the calibration process, some obvious outlier points have been eliminated. The significant differences between the types of weather are visibly evident. For free flow speed, good weather conditions experienced the highest free flow speed, which is higher than the posted speed limit $(80 \mathrm{~km} / \mathrm{h})$; under light snow conditions, the free flow speed is slightly lower than that under good weather and almost equal to the posted speed limit; when it snows heavily, the free flow speed drops drastically to $66.7 \mathrm{~km} / \mathrm{h}$. In terms of capacity, which cannot be directly observed from the FD, under good weather conditions the capacity is $1647 \mathrm{veh} / \mathrm{h} / \mathrm{l}$, while under light snow conditions the capacity is slightly lower with a number of $1572 \mathrm{veh} / \mathrm{h} / \mathrm{l}$. However, under heavy snow conditions the capacity drops as low as $1323 \mathrm{veh} / \mathrm{h} / \mathrm{l}$. In terms of critical density, the difference among the weather conditions is not as significant as the previous two parameters. Good weather conditions still show the largest critical density of $24.84 \mathrm{veh} / \mathrm{km} / \mathrm{l}$, and, under light snow conditions and heavy snow conditions, the critical densities are $22.30 \mathrm{veh} / \mathrm{km} / \mathrm{l}$ and $21.46 \mathrm{veh} / \mathrm{km} / 1$, respectively. Jam density represents the ability of a road segment to accommodate vehicles, so in this paper we assume that jam density does not change since the length and number of lanes do not change with the weather.

Table 4 shows the regression results of the weather factor parameters and statistics. The weather data used in this 


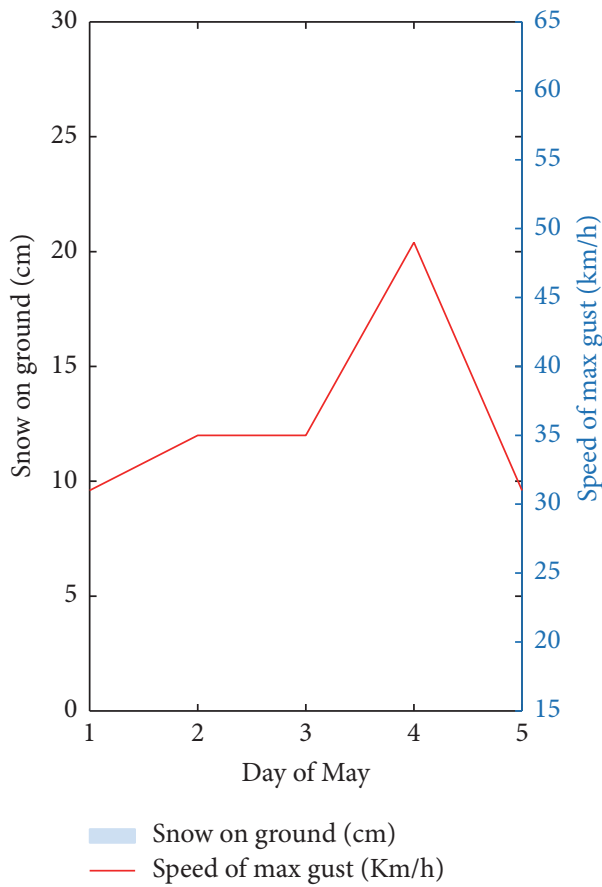

(a)

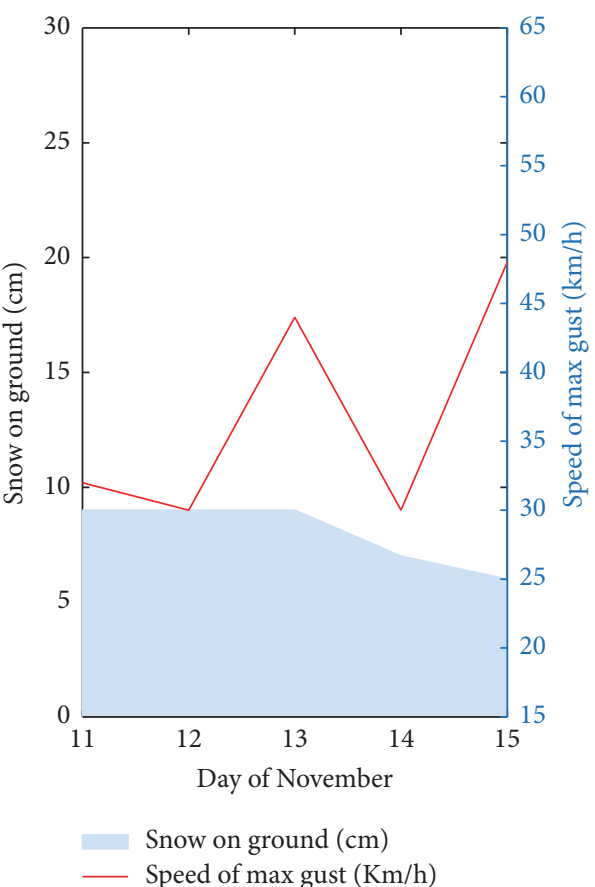

(b)

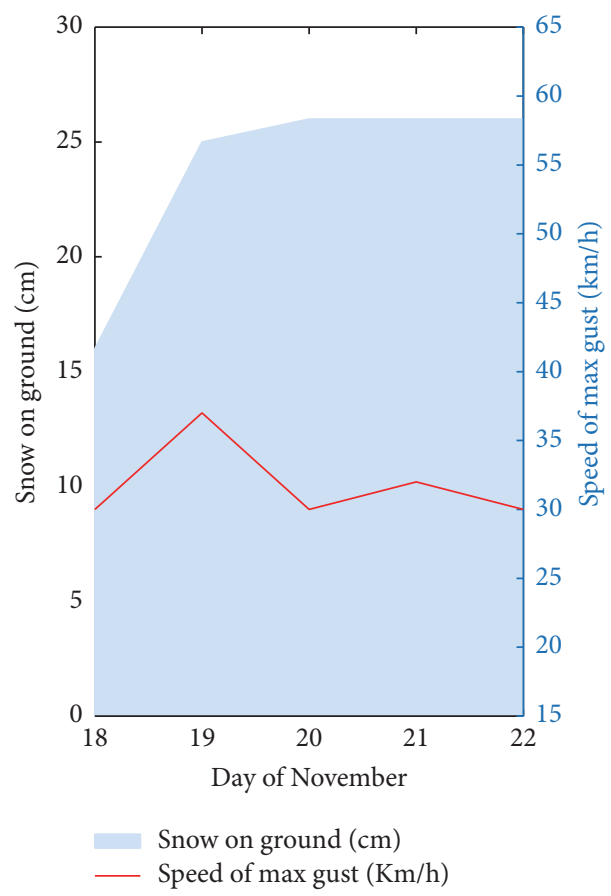

(c)

FiguRE 3: Ground snow and wind speed for the three weather conditions.

regression include the DSG and SG from May 1-5, November 11-15, and November 18-22 of 2013. Loop detector data for the same periods is also used to calibrate the FDs, and the data frequency is 20 seconds. The negative $\alpha_{1}$ indicates that capacity decreases with a positive DSG, and a positive DSG indicates that snow is accumulating and road conditions are worsening. The negative $\beta_{1}$ and $\beta_{2}$ indicate that the free flow speed will decrease with a positive value of DSG and SG, which implies that the snowfall is relatively heavy and snow is accumulating. Note that all the parameters fall within $95 \%$ confidence bounds and both regressions have a good fit; see adjusted $R$-square.

To validate the necessity of considering weather factors in the METANET prediction model, we conducted a prediction simulation, in which the seed of each calculation round was field data. We compared the prediction error of both 


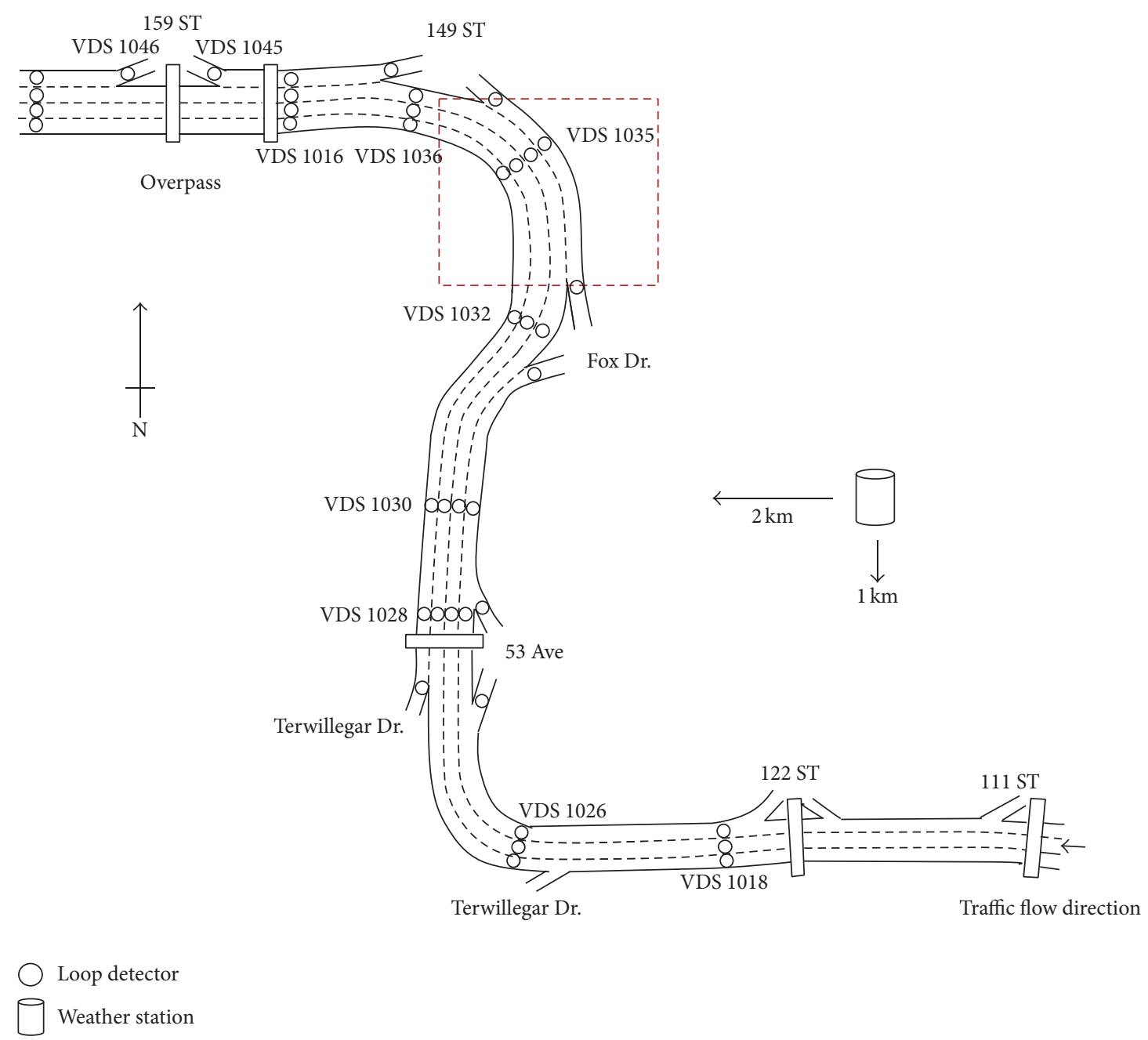

Figure 4: The location of target VDSs on Whitemud Drive, Edmonton, Canada.

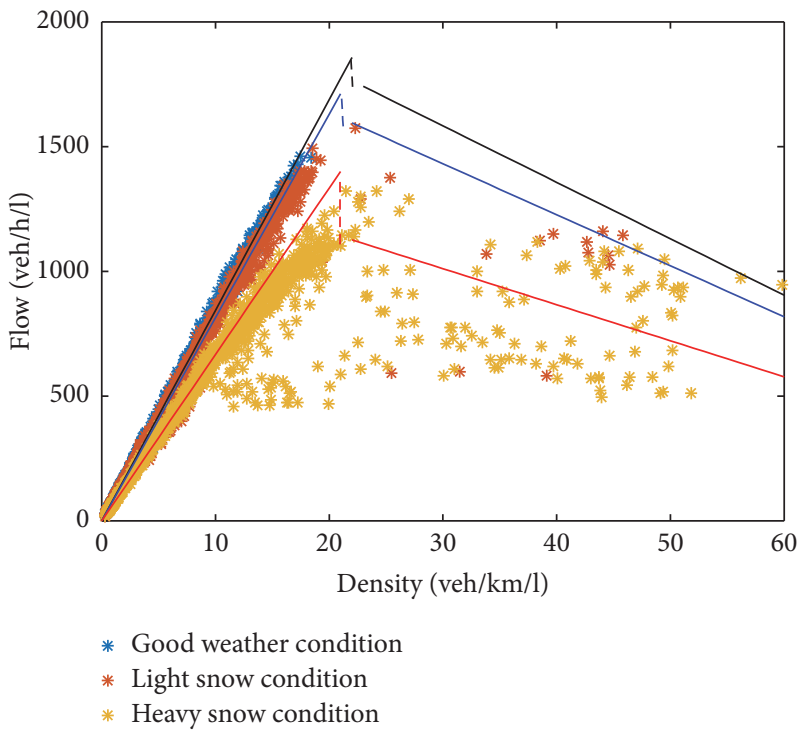

(a)

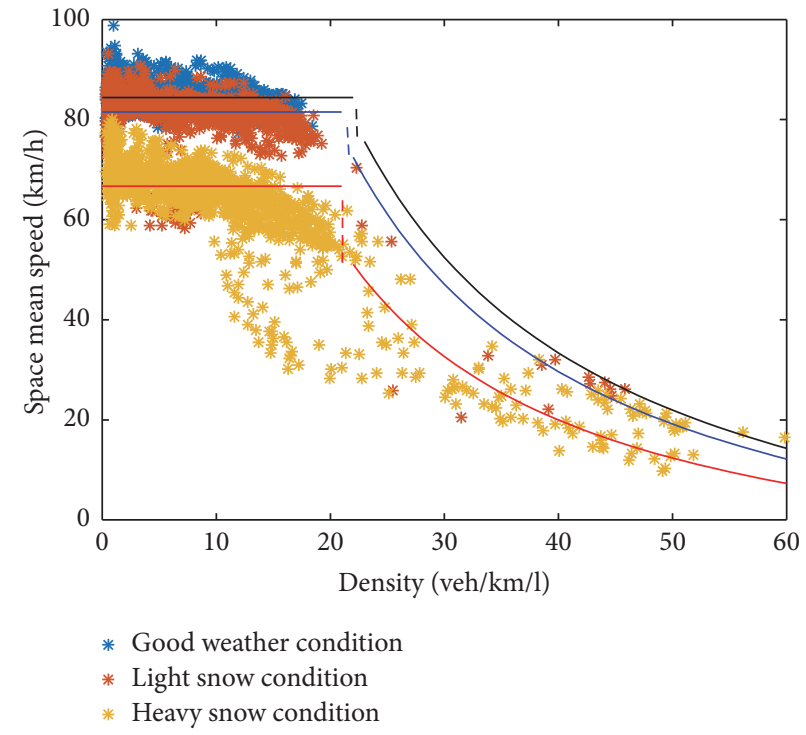

(b)

FIGURE 5: Field traffic data and calibrated FD under three weather conditions: (a) triangular form and (b) variated form. 


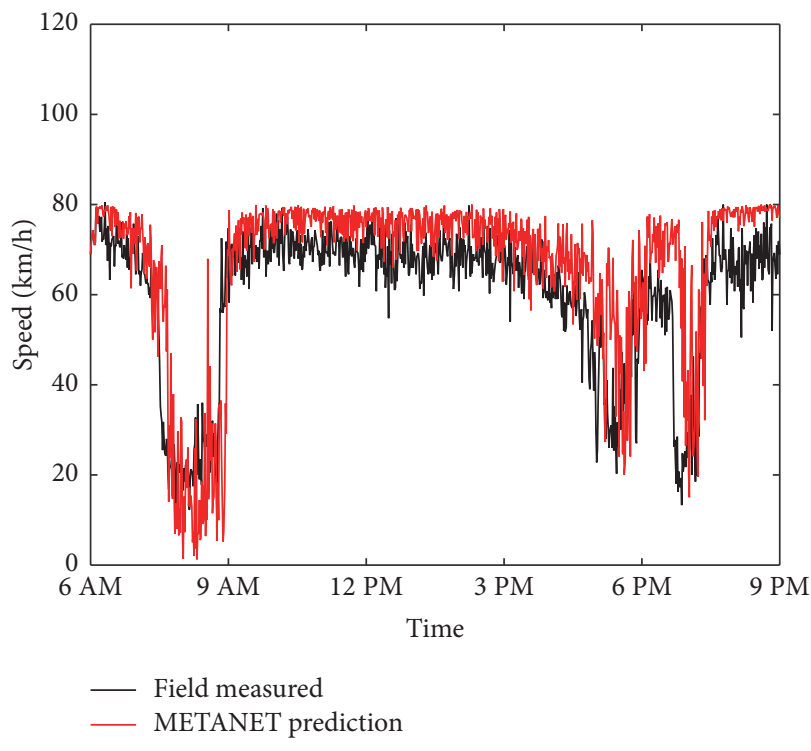

(a)

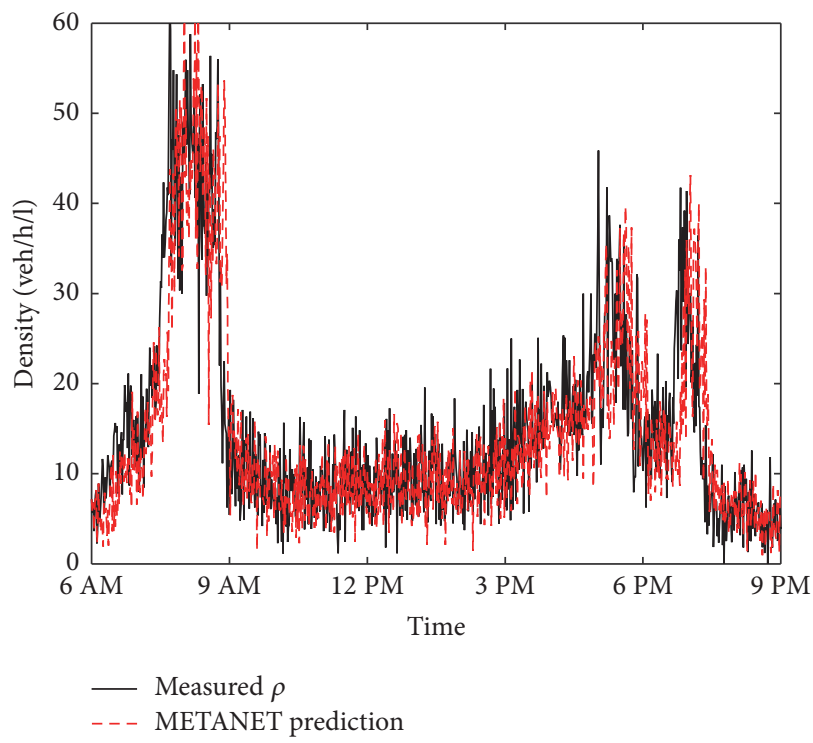

(c)

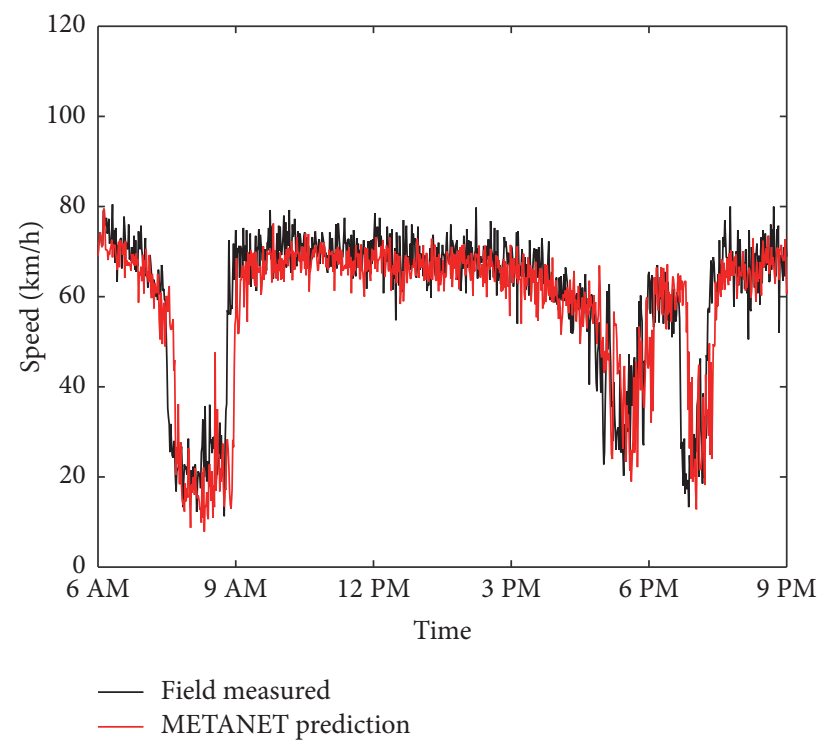

(b)

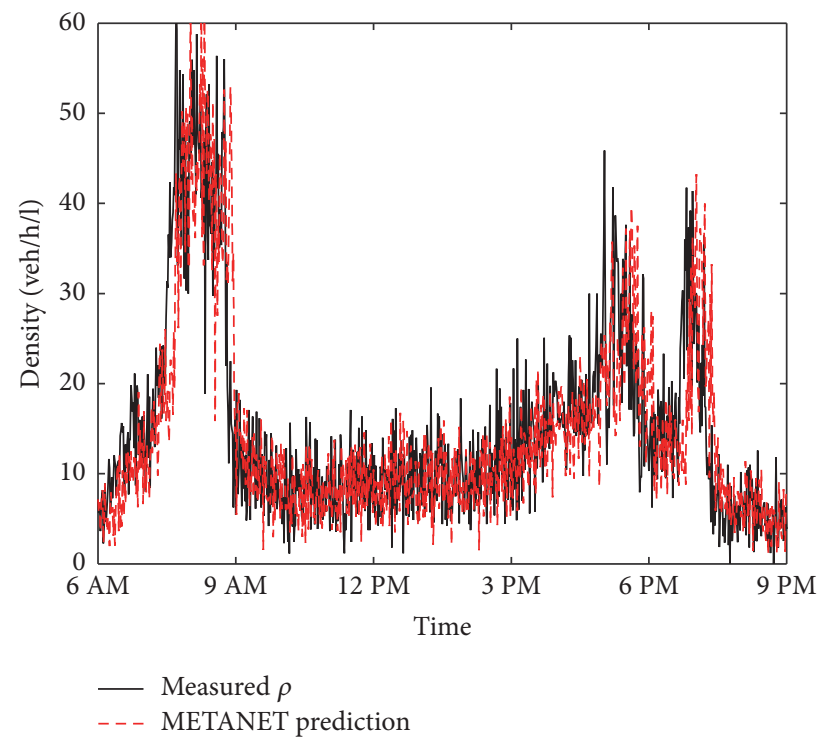

(d)

FIGURE 6: The prediction performance of average speed and density in one heavy snow day (November 18): (a) speed prediction using fixed FD parameter. (b) Speed prediction when weather factors are applied: $v_{f, i}=66.50 ; \rho_{\mathrm{cr}, i}=21.46$. (c) Density prediction using fixed FD parameter. (d) Density prediction when weather factors are applied: $v_{f, i}=66.50 ; \rho_{\mathrm{cr}, i}=21.46$.

space mean speed and density between not adding weather factor and adding weather factor conditions. The prediction scenario is set to be 10 minutes with a calculating frequency of 20 seconds with rolling horizon. The calculation interval is 20 seconds, and, in predicting the traffic status 10 minutes in advance, each round of calculation includes 30 iterations. Since the prediction simulation is not in real time, we calculate the prediction error by comparing the traffic state predicted $10 \mathrm{~min}$ in advance via METANET and the real traffic state from the field dataset. The root mean square error (RMSE) is calculated in each of the situations.

In each pair of the following three comparisons, the condition without a weather factor always uses the fixed parameters $v_{f, i}=80.06$ and $\rho_{\mathrm{cr}, i}=23.83$. Note that those fixed parameters together with other global parameters are used. These parameters indicate that, under uncongested conditions, the free flow speed can be slightly higher than the posted speed limit of $80 \mathrm{~km} / \mathrm{h}$; however, under unsatisfying weather conditions the above default setting might be too high. Based on the weather-specific FD assumption, we apply the calibrated weather factors to $v_{f, i}$ and $\rho_{\mathrm{cr}, i}$, while other global parameters of METANET remain unchanged. From each type of weather, we picked up one day as a case to conduct a prediction experiment for both speed and density variables. For heavy snow conditions, we chose November 18 (Figure 6); for light snow conditions, we chose November 14 


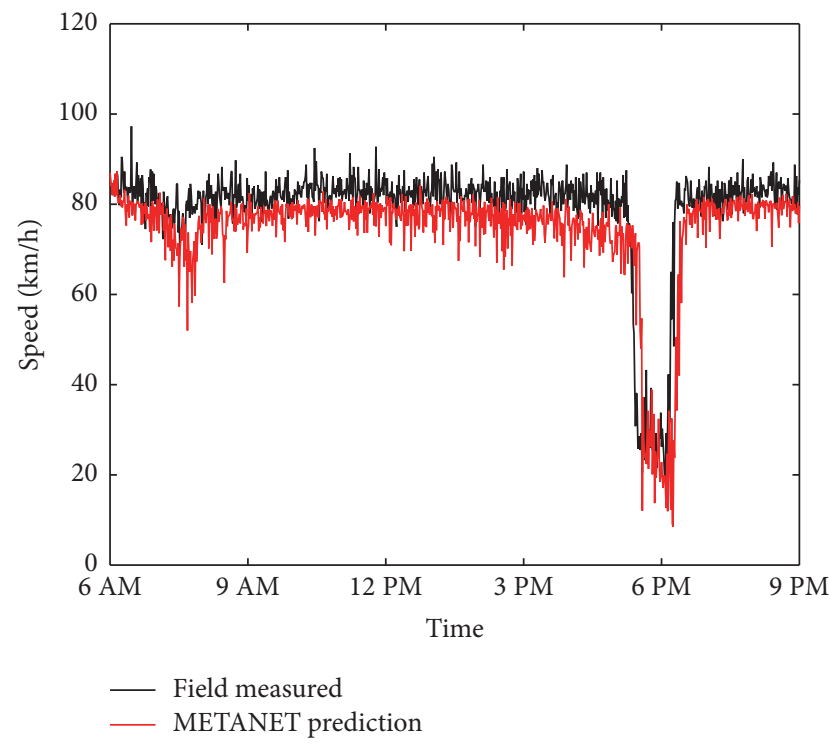

(a)

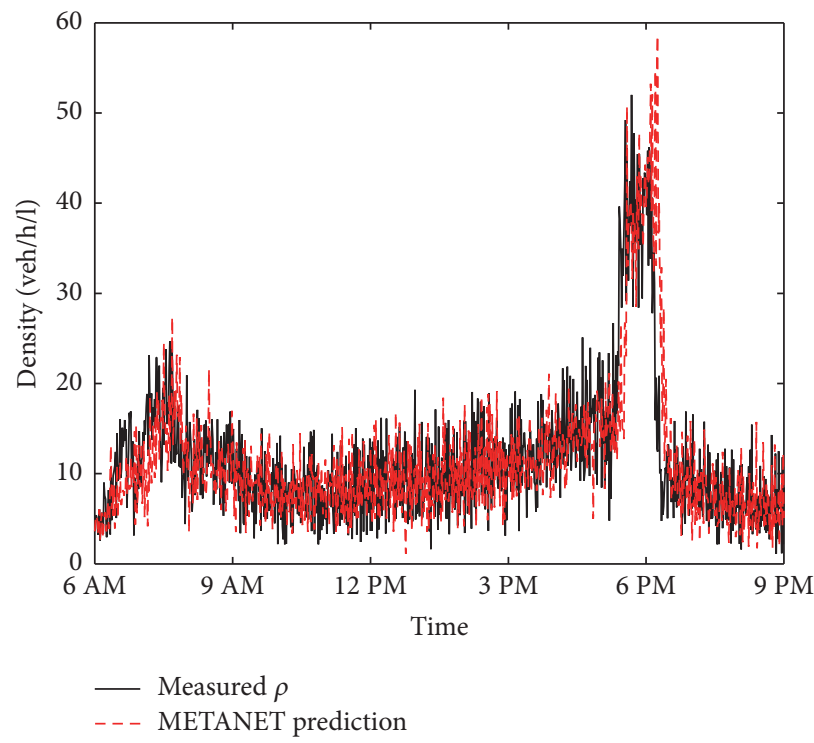

(c)

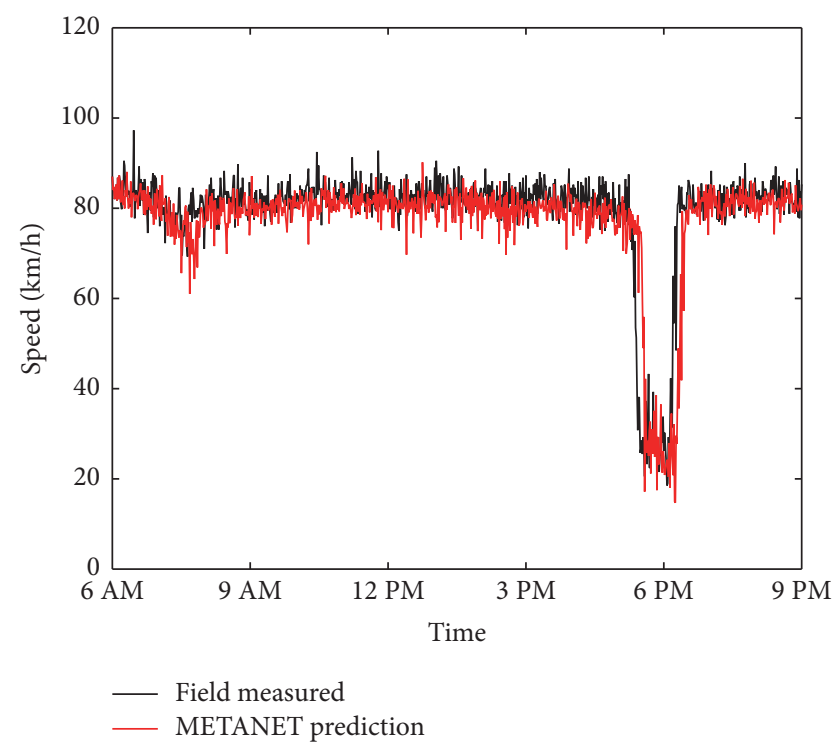

(b)

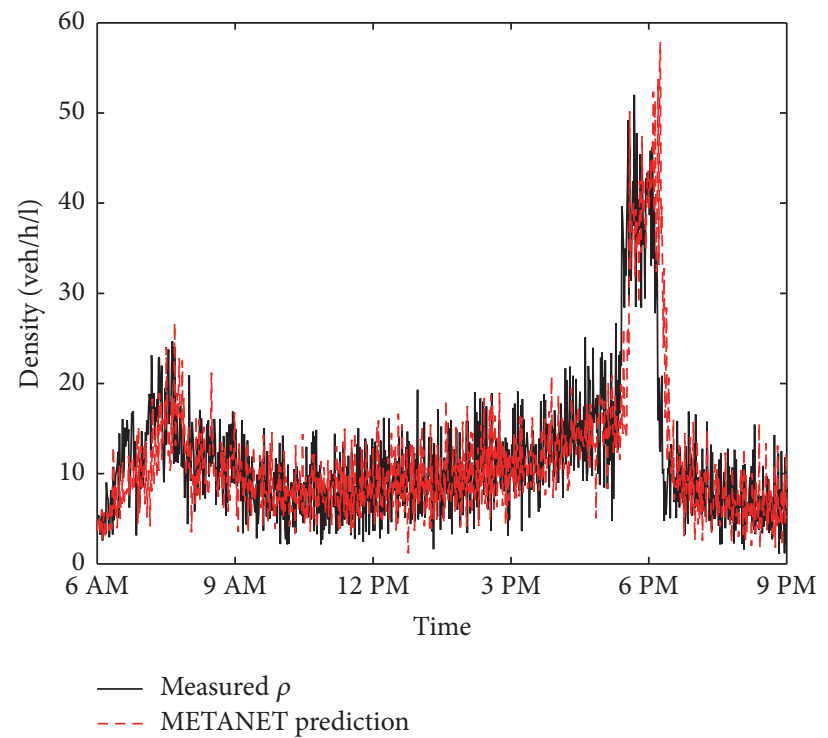

(d)

FIGURE 7: The prediction performance of average speed and density in one light snow day (November 14): (a) speed prediction using fixed FD parameter. (b) Speed prediction when weather factors are applied: $v_{f, i}=81.08 ; \rho_{\mathrm{cr}, i}=22.70$. (c) Density prediction using fixed FD parameter. (d) Density prediction when weather factors are applied: $v_{f, i}=81.08 ; \rho_{\mathrm{cr}, i}=22.70$.

(Figure 7); and, for good weather, we chose May 2 (Figure 8). For each day the traffic state prediction ran from $6 \mathrm{AM}$ to 9 PM. In Figures 6-8, (a) and (b) compare the prediction performance of average speed with and without adding weather factors. (c) and (d) of Figures 6-8 compare the prediction performance of traffic density with and without adding weather factors. The prediction result of traffic flow is not shown since it can be easily calculated through flow identical equation (8). The fixed global parameter values are shown in Table 5. Those global parameters are calibrated using the traffic data of the first day of November, 2013.

It is found from Figures 6-8 that in speed prediction simulation observable prediction error can be seen while the
TABLE 5: Calibrated METANET global parameters.

\begin{tabular}{lccc}
\hline$\tau$ & $\eta$ & $\kappa$ & $\alpha$ \\
\hline 120.00 & 37.98 & 10.00 & 2.29 \\
\hline
\end{tabular}

error in density prediction is invisible. The reason is that the speed dynamics model heavily relies on the parameters, while density dynamics are simply derived from mass conservation. Tables 6 and 7 show detailed quantitative error measurement for comparison. Under heavy snow conditions, the whole daytime speed RMSE of conventional prediction is 11.69, while in weather-specific prediction the RMSE is 9.52. During 


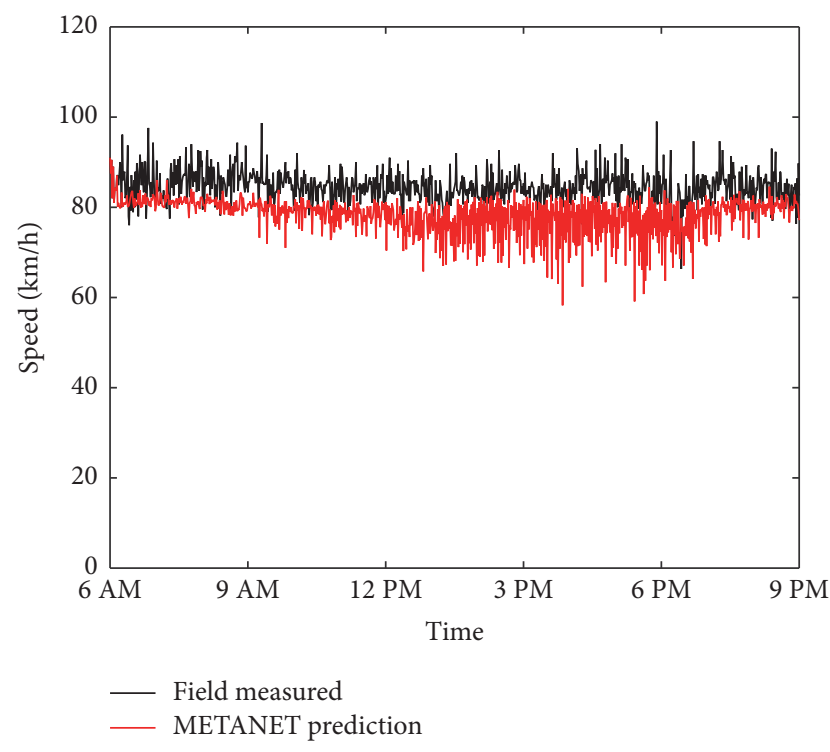

(a)

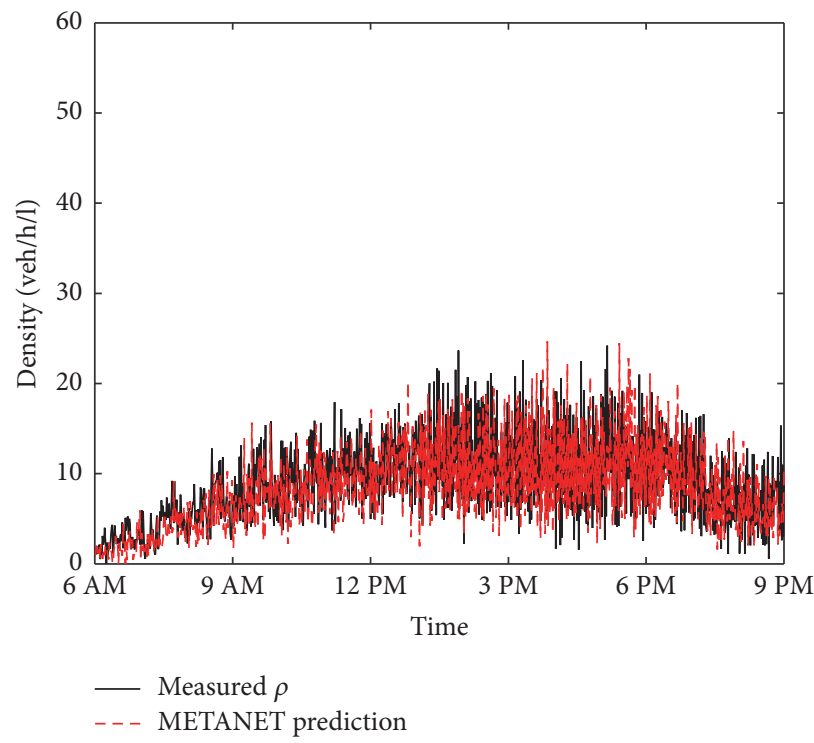

(c)

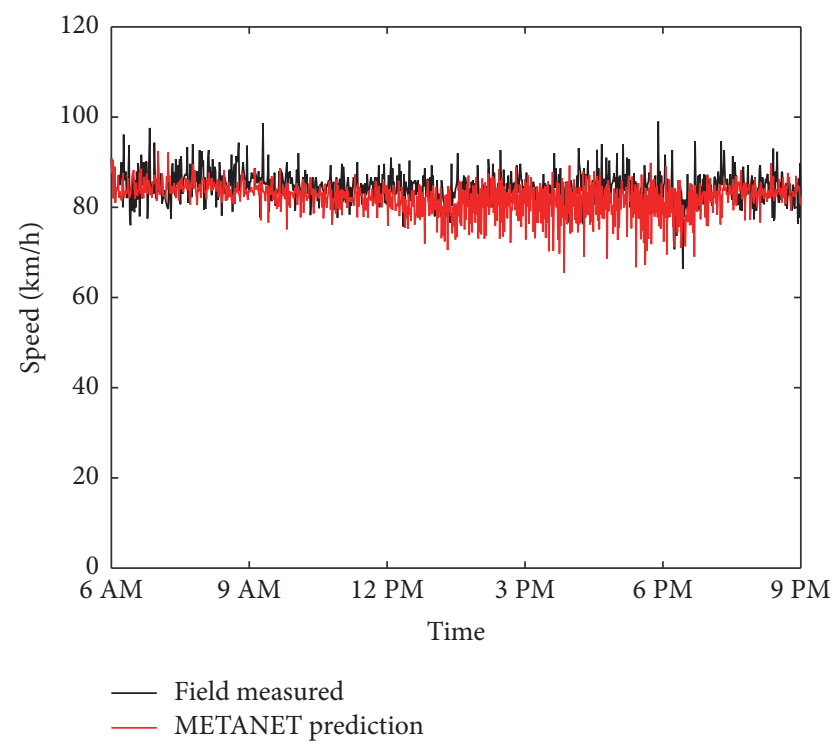

(b)

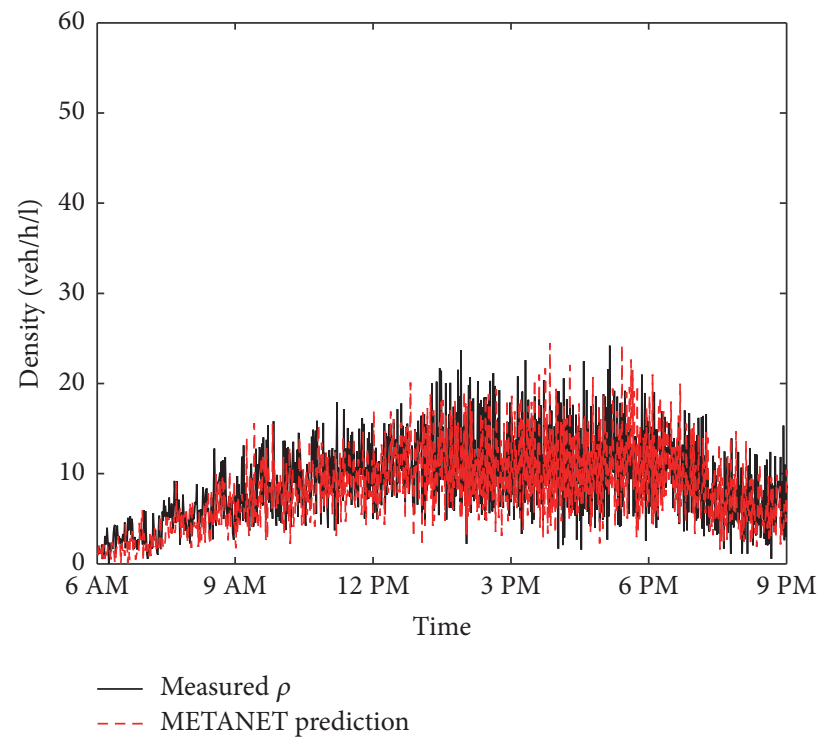

(d)

FIGURE 8: The prediction performance of average speed and density in one good weather day (May 02): (a) speed prediction using fixed FD parameter. (b) Speed prediction when weather factors are applied: $v_{f, i}=84.06 ; \rho_{\mathrm{cr}, i}=23.83$. (c) Density prediction using fixed FD parameter. (d) Density prediction when weather factors are applied: $v_{f, i}=84.06 ; \rho_{\mathrm{cr}, i}=23.83$.

TABLE 6: Speed prediction root mean square error (RMSE) $(\mathrm{km} / \mathrm{h})$.

\begin{tabular}{lcccc}
\hline \multicolumn{1}{c}{ Feature: RMSE of speed prediction } & Good weather condition & Light snow condition & Heavy snow condition \\
\hline Daytime & Conventional & 6.88 & 9.12 & 11.69 \\
6 AM-9 PM & Weather-specific & $\mathbf{5 . 3 2}$ & $\mathbf{8 . 2 1}$ & $\mathbf{9 . 5 2}$ \\
AM peak hours & Conventional & 6.47 & 8.35 & 16.21 \\
7 AM-9 PM & Weather-specific & $\mathbf{4 . 5 4}$ & $\mathbf{6 . 8 1}$ & $\mathbf{1 4 . 9 6}$ \\
PM peak hours & Conventional & 9.39 & 15.89 & 17.43 \\
4 PM-7 PM & Weather-specific & $\mathbf{6 . 4 5}$ & $\mathbf{1 5 . 2 9}$ & $\mathbf{1 3 . 5 1}$ \\
\hline
\end{tabular}


TABLE 7: Density prediction root mean square error (RMSE) (veh/km/l).

\begin{tabular}{lccc}
\hline Feature: RMSE of density prediction & Good weather condition & Light snow condition & Heavy snow condition \\
\hline Conventional prediction & 4.46 & 6.02 & 7.17 \\
Weather-specific prediction & $\mathbf{4 . 4 5}$ & $\mathbf{5 . 9 8}$ & $\mathbf{7 . 1 4}$ \\
\hline
\end{tabular}

PM peak hours, the RMSE dropped drastically from 17.43 to 13.51, and the same situation applies to AM peak hours. Under light snow conditions and good weather conditions, the whole daytime speed RMSE of conventional prediction is 9.12, while in weather-specific prediction the RMSE is 8.21. During AM peak hours, the RMSE drastically dropped from 8.35 to 6.81 . Under good weather conditions, the whole daytime speed RMSE for conventional prediction is 6.88, while in the weather-specific prediction the RMSE is 5.32 . During PM peak hours the RMSE dropped from 9.39 to 6.45, which is more drastic than daytime average and AM peak hours; however, under good weather conditions, the original speed RMSE is small enough. In terms of density prediction, due to the nature of the density dynamics, the conventional prediction and weather-specific prediction did not show significant difference. The RMSE difference range 0.01 0.03 may be attributed to random computation error. Thus, the prediction error of flow is proportional to the prediction error of speed. It can be concluded that the weather-specific METANET prediction will be more helpful under the most adverse weather conditions and during peak hours when speed drops steeply.

\section{Conclusions}

Various kinds of traffic flow models are used to describe traffic flow characteristics; however, very few of them describe the explicit negative impact of adverse weather on travel speed, flow capacity, critical density, and many other aspects, such as driving safety. This paper demonstrated that weather conditions indeed impact the driving environment and driver behavior so that it is necessary to build weather-specific FDs. The proposed weather factor modelling is one possible way.

The modelling of weather factors in this paper is reasonable, practical, and repeatable. The same weather variable filtering method can be applied to other cases if researchers have access to higher resolution weather data, such as hourly weather data, or data on other weather events, such as rain and fog. Using the same method, researchers can assess different significant variables according to the situation. Through prediction simulation, macroscopic traffic prediction accuracy is found to be improved after introducing weatherspecific FD parameters, especially in speed prediction. In adverse weather conditions, weather factors improved the accuracy of macroscopic traffic prediction models. This study is limited by the accessibility to higher resolution of weather data and future studies may propose more sophisticated weather modelling method. Future studies may also look into the combination of a weather-specific FD and real-time weather forecast and consider their implementation into realtime traffic control.

\section{Notations}

$T: \quad$ Data collection interval $=20$ seconds $(\mathrm{s})$

$\Delta_{i}: \quad$ Length of section $i(\mathrm{~km})$

$\Omega: \quad$ Set of space and time $(x, t)$

$q(x, t): \quad$ Traffic flow at time $t$, space $x(\mathrm{veh} / \mathrm{h})$

$\rho(x, t): \quad$ Traffic density at time $t$, space $x(\mathrm{veh} / \mathrm{km})$

$v(x, t): \quad$ Vehicle space mean speed at time $t$, space $x(\mathrm{~km} / \mathrm{h})$

$r(t, x): \quad$ On-ramp flow at time $t$, space $x(\mathrm{veh} / \mathrm{h})$

$s(t, x): \quad$ Off-ramp flow at time $t$, space $x(\mathrm{veh} / \mathrm{h})$

$q_{i}(k): \quad$ Traffic flow entering section $i$ at time step $k(\mathrm{veh} / \mathrm{h})$

$\rho_{i}(k): \quad$ Traffic density at time step $k$, section $i(\mathrm{veh} / \mathrm{km})$

$v_{i}(k): \quad$ Space mean speed at time step $k$, section $i(\mathrm{~km} / \mathrm{h})$

$r_{i}(k): \quad$ On-ramp flow at time step $k$, section $i(\mathrm{veh} / \mathrm{h})$

$s_{i}(k): \quad$ Off-ramp flow at time step $k$, section $i(\mathrm{veh} / \mathrm{h})$

$V\left(\rho_{i}(k)\right)$ : Desired speed in speed dynamics in the METANET model $(\mathrm{km} / \mathrm{h})$

$\Theta: \quad$ Set of global parameters of METANET model

$C_{i}: \quad$ Flow capacity of FD at section $i(\mathrm{veh} / \mathrm{h})$

$\rho_{\mathrm{cr}, i}: \quad$ Critical density of FD at section $i(\mathrm{veh} / \mathrm{km})$

$\rho_{\mathrm{jam}, i}: \quad$ Jam density of FD at section $i(\mathrm{veh} / \mathrm{km})$

$v_{f, i}: \quad$ Free flow speed of FD at section $i(\mathrm{~km} / \mathrm{h})$

$\theta_{i}: \quad$ Capacity drop fraction of FD at section $i$

$w^{v_{f}}$ : Weather adjustment factor for free flow speed of FD

$w^{C}: \quad$ Weather adjustment factor for flow capacity of FD

$w^{\rho_{c r}}: \quad$ Weather adjustment factor for critical density of FD

SG: $\quad$ Snow on the ground $(\mathrm{cm})$

DSG: Derivation of snow on the ground $(\mathrm{cm})$.

\section{Disclosure}

The contents of this paper reflect the views of the authors who are responsible for the facts and the accuracy of the data presented herein. The contents do not necessarily reflect the official views or policies of the City of Edmonton and Transport Canada. This paper does not constitute a standard, specification or regulation.

\section{Competing Interests}

The authors declare that there is no conflict of interests regarding the publication of this paper. 


\section{Acknowledgments}

The authors would like to thank Wai Cheung, Janis Chow, Michael Vaudan, Adrian Loh, and Rahim Karmali from the traffic operation group in the City of Edmonton for providing the Whitemud Drive loop detector data. This research work was jointly supported by the Natural Sciences and Engineering Research Council (NSERC) of Canada, City of Edmonton, and Transport Canada. The author would also like to thank the National Natural Science Foundation of China (no. 51578432) for sponsoring publication process of this paper.

\section{References}

[1] B. M. Williams and L. A. Hoel, "Modeling and forecasting vehicular traffic flow as a seasonal ARIMA process: theoretical basis and empirical results," Journal of Transportation Engineering, vol. 129, no. 6, pp. 664-672, 2003.

[2] C. Antoniou, M. Ben-Akiva, and H. N. Koutsopoulos, "Online calibration of traffic prediction models," Transportation Research Record, vol. 1934, pp. 235-245, 2005.

[3] S. Afandizadeh and J. Kianfar, "A hybrid neuro-genetic approach to short-term traffic volume prediction," International Journal of Civil Engineering, vol. 7, no. 1, pp. 41-48, 2009.

[4] B. L. Smith, B. M. Williams, and R. Keith Oswald, "Comparison of parametric and nonparametric models for traffic flow forecasting," Transportation Research Part C: Emerging Technologies, vol. 10, no. 4, pp. 303-321, 2002.

[5] J. Laval, Z. He, and F. Castrillon, "Stochastic extension of Newell's three-detector method," Transportation Research Record, vol. 2315, pp. 73-80, 2012.

[6] A. Spiliopoulou, M. Kontorinaki, M. Papageorgiou, and P. Kopelias, "Macroscopic traffic flow model validation at congested freeway off-ramp areas," Transportation Research Part C: Emerging Technologies, vol. 41, pp. 18-29, 2014.

[7] M. J. Lighthill and G. B. Whitham, "On kinematic waves. II. A theory of traffic flow on long crowded roads," Proceedings of the Royal Society of London A. Mathematical, Physical and Engineering Science, vol. 229, pp. 317-345, 1955.

[8] P. I. Richards, "Shock waves on the highway," Operations Research, vol. 4, pp. 42-51, 1956.

[9] C. F. Daganzo, "The cell transmission model: a dynamic representation of highway traffic consistent with the hydrodynamic theory," Transportation Research Part B: Methodological, vol. 28, no. 4, pp. 269-287, 1994.

[10] C. F. Daganzo, "A behavioral theory of multi-lane traffic flow. Part I: long homogeneous freeway sections," Transportation Research Part B: Methodological, vol. 36, no. 2, pp. 131-158, 2002.

[11] C. F. Daganzo, "A behavioral theory of multi-lane traffic flow. Part II: merges and the onset of congestion," Transportation Research Part B: Methodological, vol. 36, no. 2, pp. 159-169, 2002.

[12] H. J. Payne, "Models of freeway traffic and control," in Mathematical Models of Public Systems: Simulation Council Proceedings Series, pp. 51-61, 1971.

[13] J. A. Laval and C. F. Daganzo, "Lane-changing in traffic streams," Transportation Research Part B: Methodological, vol. 40, no. 3, pp. 251-264, 2006.

[14] Y. Wang and M. Papageorgiou, "Real-time freeway traffic state estimation based on extended Kalman filter: a general approach," Transportation Research Part B: Methodological, vol. 39, no. 2, pp. 141-167, 2005.

[15] L. Mihaylova, R. Boel, and A. Hegyi, "Freeway traffic estimation within recursive Bayesian framework," Automatica, vol. 43, no. 2, pp. 290-300, 2007.

[16] J. C. Herrera and A. M. Bayen, "Incorporation of Lagrangian measurements in freeway traffic state estimation," Transportation Research Part B: Methodological, vol. 44, no. 4, pp. 460-481, 2010.

[17] G. F. Newell, "A simplified theory of kinematic waves in highway traffic, part II: queueing at freeway bottlenecks," Transportation Research Part B, vol. 27, no. 4, pp. 289-303, 1993.

[18] G. F. Newell, "A simplified theory of kinematic waves in highway traffic I: general theory. II: queuing at freeway bottlenecks. III: multi-destination flows," Transportation Research Part B, vol. 27, pp. 281-313, 1993.

[19] M. Papageorgiou, J.-M. Blosseville, and H. Hadj-Salem, "Macroscopic modelling of traffic flow on the Boulevard Périphérique in Paris," Transportation Research Part B, vol. 23, no. 1, pp. 29-47, 1989.

[20] M. Papageorgiou, H. Hadj-Salem, J. M. Blosseville, and N. Bhouri, Control, Computers, Communications in Transportation, Elsevier, 1990.

[21] Y. Bie, Implementing model predictive control based variable speed limit on urban freeways: data imputation, model modification and field test analysis [Ph.D. dissertation], University of Alberta, 2016.

[22] A. Hegyi, M. Burger, B. De Schutter, J. Hellendoorn, and T. J. J. Van Den Boom, "Towards a practical application of model predictive control to suppress shock waves on freeways," in Proceedings of the European Control Conference (ECC '07), vol. 19, pp. 1764-1771, July 2007.

[23] T. H. Maze, M. Agarwal, and G. Burchett, "Whether weather matters to traffic demand, traffic safety, and traffic operations and flow," Transportation Research Record, no. 1948, pp. 170-176, 2006.

[24] J. Asamer and M. Reinthaler, "Estimation of road capacity and free flow speed for urban roads under adverse weather conditions," in Proceedings of the 13th International IEEE Conference on Intelligent Transportation Systems (ITSC 2010), pp. 812-818, Funchal, Portugal, September 2010.

[25] HCM, Highway capacity manual 2010, Transportation Research Board, National Research Council, 2010.

[26] T.-J. Kwon, L. Fu, and C. Jiang, "Effect of winter weather and road surface conditions on macroscopic traffic parameters," in Proceedings of the Transportation Research Board Annual Meeting, vol. 862, p. 16, 2013.

[27] L. Zhang, P. Holm, and J. Colyar, "Identifying and assessing key weather-related parameters and their impacts on traffic operations using simulation," Federal Highway Administration Report FHWA-HRT-04-131, 2004.

[28] H. Rakha, M. Farzaneh, M. Arafeh, R. Hranac, E. Sterzin, and D. Krechmer, "Empirical studies on traffic flow in inclement weather," Final Report-Phase I 107, 2007.

[29] H. Alhassan and J. Ben-Edigbe, "Extent of highway capacity loss due to rainfall," International Journal of Civil, Environmental, Structural, Construction and Architectural Engineering, vol. 6, no. 12, pp. 1154-1161, 2012.

[30] T. Hou, H. Mahmassani, R. Alfelor, J. Kim, and M. Saberi, "Calibration of traffic flow models under adverse weather and application in mesoscopic network simulation procedures," 
Transportation Research Record: Journal of the Transportation Research Board, vol. 2, no. 2391, pp. 92-104, 2013.

[31] U. Shahdah and L. Fu, Quantifying the Mobility Benefits of Winter Road Maintenance-A Simulation-Based Analysis, 2010.

[32] W. H. K. Lam, M. Asce, M. L. Tam, X. Cao, and X. Li, "Modeling the effects of rainfall intensity on traffic speed, flow, and density relationships for urban roads," Journal of Transportation Engineering, vol. 139, no. 7, pp. 758-770, 2013.

[33] A. Messmer and M. Papageorgiou, "METANET: a macroscopic simulation program for motorway networks," Traffic Engineering \& Control, vol. 31, no. 8-9, pp. 466-470, 1990.

[34] P. K. Munjal and L. A. Pipes, "Propagation of on-ramp density perturbations on unidirectional two- and three-lane freeways," Transportation Research, vol. 5, no. 4, pp. 241-255, 1971.

[35] K. Pearson, "Note on regression and inheritance in the case of two parents," Proceedings of the Royal Society of London, vol. 58, pp. 240-242, 1895.

[36] S. M. Stigler, "Francis Galton's account of the invention of correlation," Statistical Science, vol. 4, no. 2, pp. 73-79, 1989. 


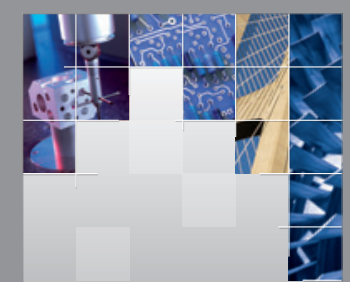

\section{Enfincering}
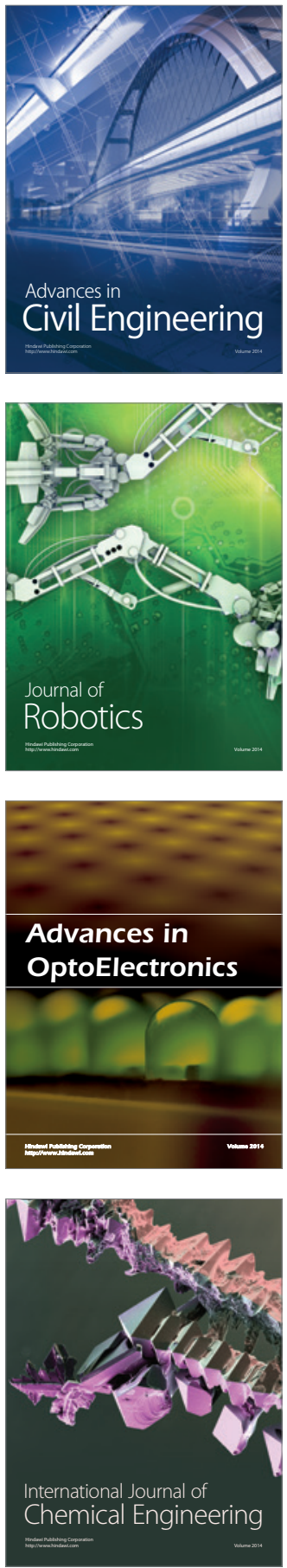

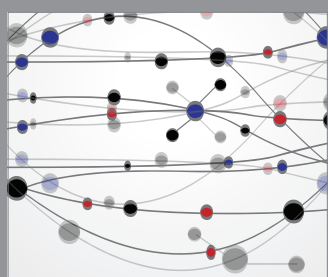

The Scientific World Journal

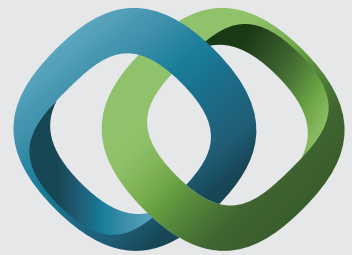

\section{Hindawi}

Submit your manuscripts at

https://www.hindawi.com
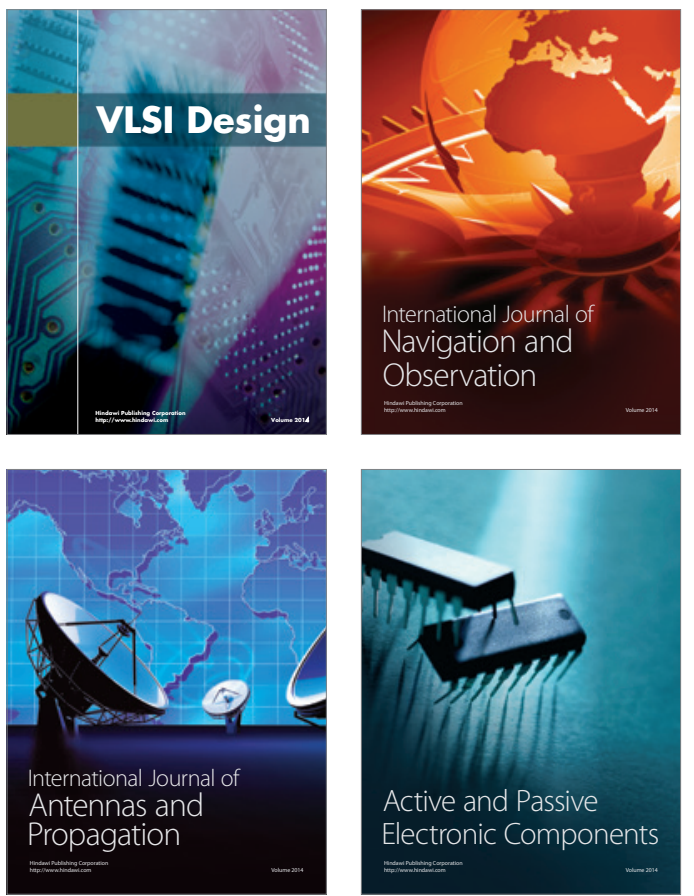
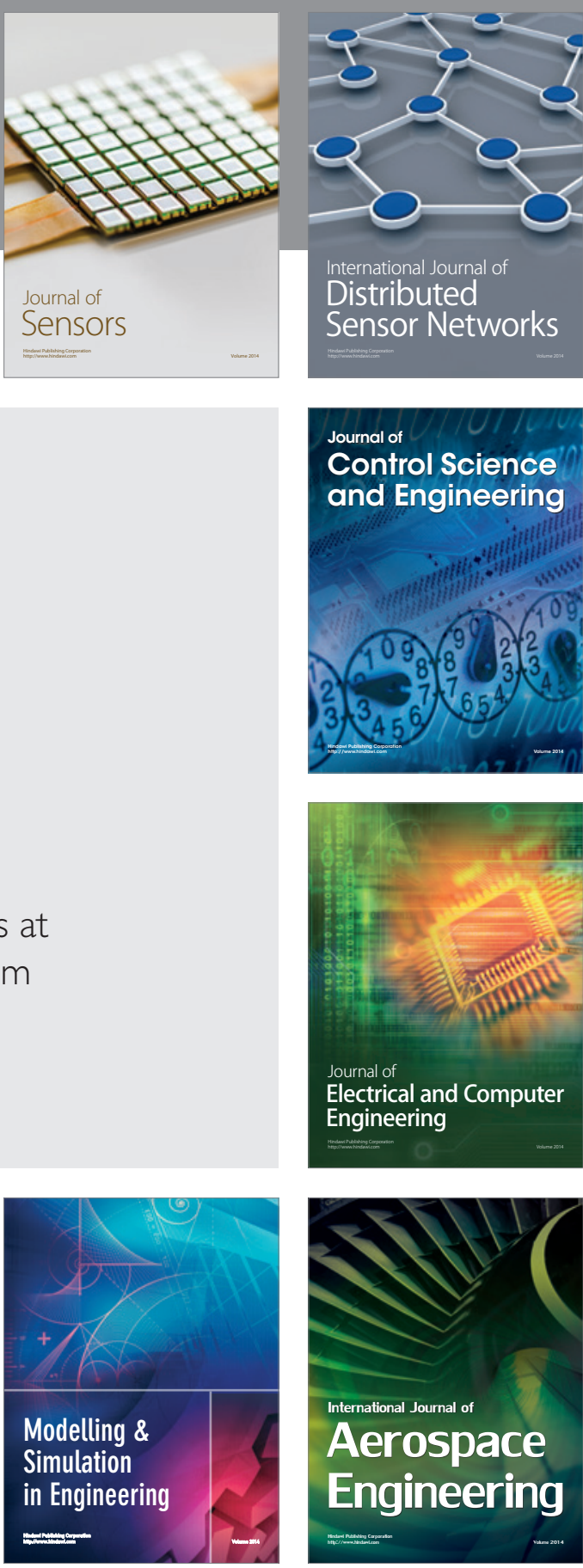

International Journal of

Distributed

Sensor Networks

$-$

Joumal of

Control Science

and Engineering
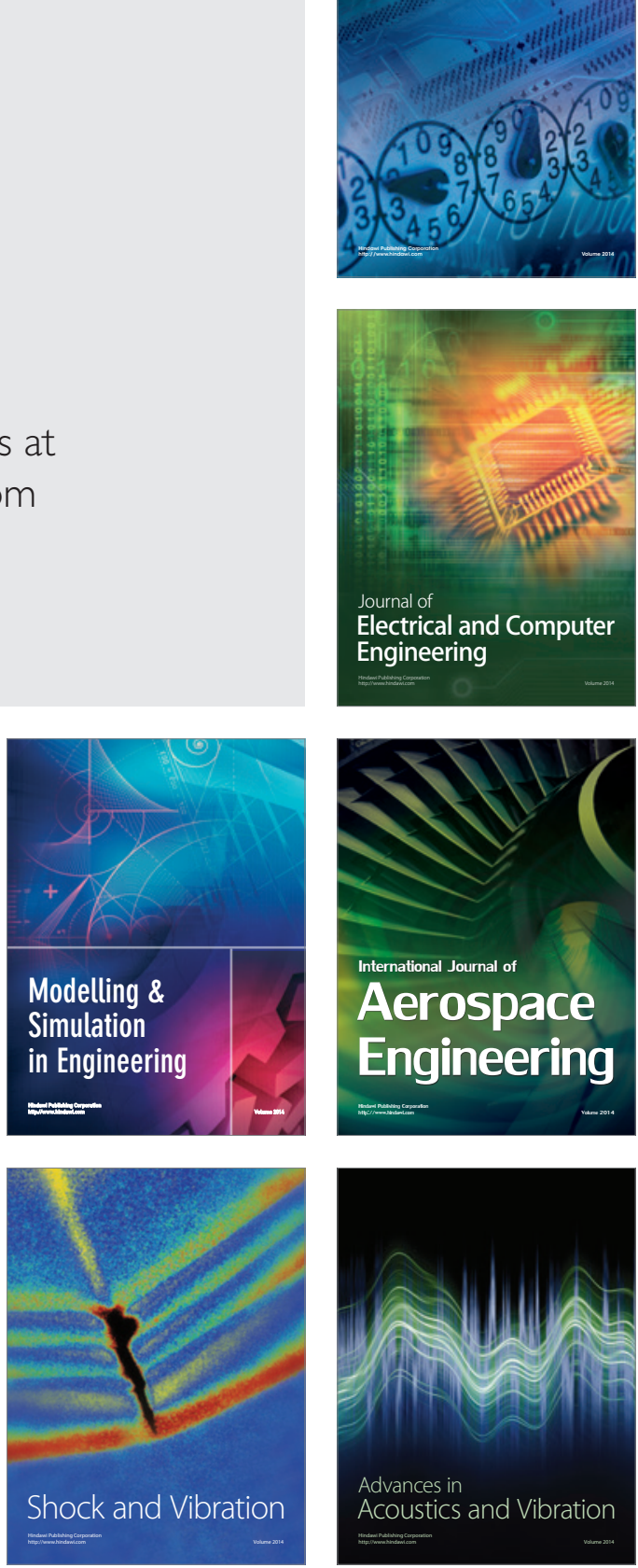